\title{
SWARM-BOT: From Concept to Implementation
}

\author{
Francesco Mondada, André Guignard, Michael Bonani, Daniel Bär, Michel Lauria, Dario Floreano \\ Autonomous Systems Lab (http://asl.epfl.ch) \\ Swiss Federal Institute of Technology in Lausanne (EPFL) \\ CH-1015 Lausanne, Switzerland \\ email: firstname.lastname@epfl.ch
}

\begin{abstract}
This paper presents a new robotic concept, called SWARM-BOT, based on a swarm of autonomous mobile robots with self-assembling capabilities. SWARM-BOT takes advantage from collective and distributed approaches to ensure robustness to failures and to hard environment conditions in tasks such as navigation, search and transportation in rough terrain. One SWARM-BOT is composed of a number of simpler robots, called s-bots, physically interconnected. The SWARM-BOT is provided with selfassembling and self-reconfiguring capabilities whereby s-bots can connect and disconnect forming large flexible structures. This paper introduces the SWARM-BOT concept and describes its implementation from a mechatronic perspective.
\end{abstract}

\section{INTRODUCTION}

Robust, all terrain, flexible operation is interesting in applications like semi-automatic space exploration [17], rescue [6] or underwater exploration [3]. Despite international research effort in rover design [9], [17], [15], [4] and self-reconfigurable robots (overview in [11], [18]), the combination of flexibility, robustness and all-terrain navigation, even if semi-autonomous, is still difficult to achieve.

Biological systems, on the other hand, combine very well these features and show interesting examples of self-organizing structures able to deal with very different complex tasks. Social insects, in particular, show ability to self-organize in colonies which can solve problems of navigation and transportation unfeasible for a single individual [12]. This is the case of ants building bridges between branches of a tree, building rafts to survive inundations or bridges over rivers (see overview in [2]).

The goal of the SWARM-BOTS project ${ }^{1}$ is to take inspiration from ant self-assembling mechanisms and structures and translate some features into a robotic system. The project includes design, hardware implementation, test and use of this type of self-assembling, self-organizing, metamorphic robotic systems. An important part of the project consists in the physical construction of at least one SWARM-BOT, that is, a self-assembling and selforganizing robot colony composed of a number (30-35) of smaller devices, called s-bots, physically interconnected.

1http://www.swarm-bots.org

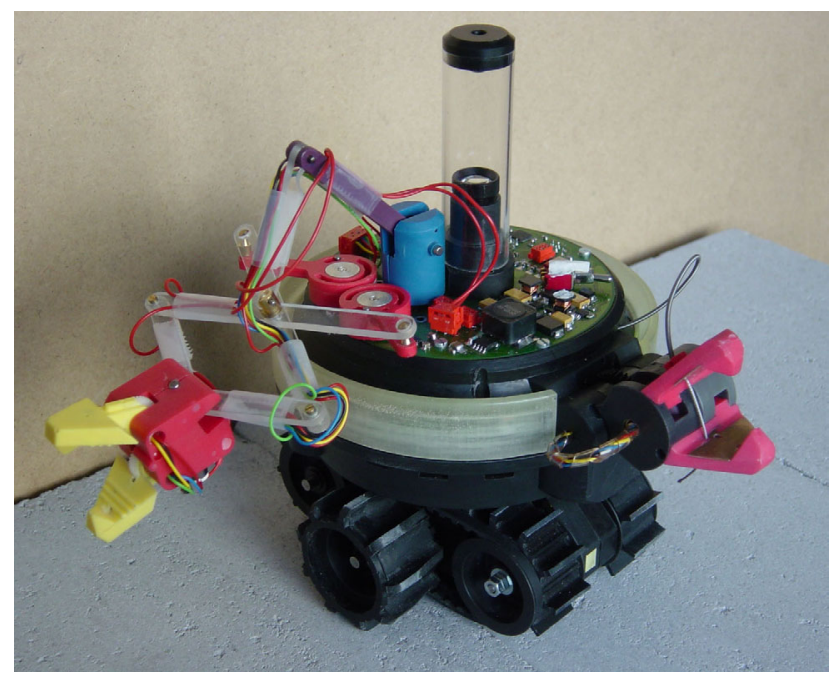

Fig. 1. One of the s-bot prototypes. The diameter of the main body is $116 \mathrm{~mm}$. A SWARM-BOT is composed by a colony of approximately 30 s-bots.

The next section describes the SWARM-BOT concept, showing the particular features of this system. Section 3 focuses on the prototype implementation, illustrating design choices and original solutions. A conclusion is given in the last section.

\section{SWARM-BOT CONCEPT}

A SWARM-BOT entity is composed of many (2 to $35)$ single robots (s-bots) physically interconnected. Each s-bot is a fully autonomous mobile robot capable of performing basic tasks such as autonomous navigation, perception of the environment and grasping of objects. In addition to these features, an s-bot is able to communicate with other s-bots and physically connect to them in flexible ways, thus forming a SWARM-BOT. The SWARM-BOT is able to perform exploration, navigation and transport of heavy objects in very rough terrain, where a single sbot has major problems to achieve the task. This hardware structure is combined with a distributed adaptive control architecture (general approach see [10], applied to SWARM-BOT see [8]) inspired upon ant colony behaviors [12]. Figure 2 shows some views of an s-bot prototype. 

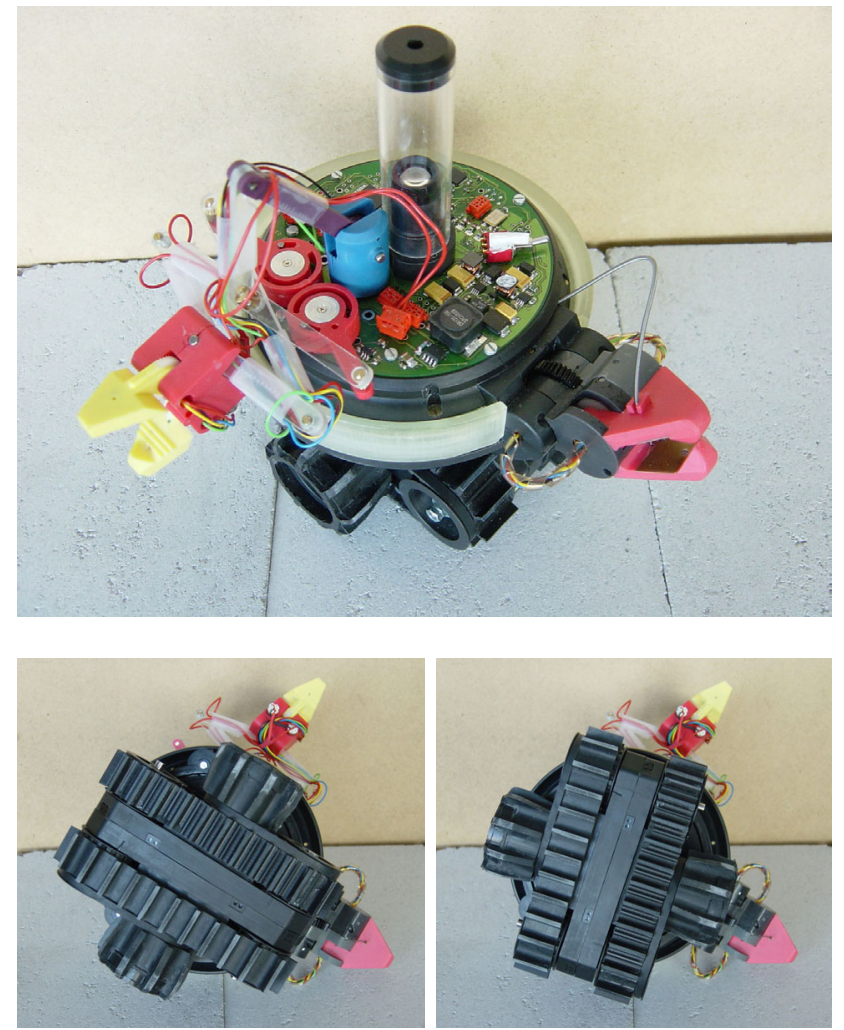

Fig. 2. Top and bottom view of the s-bot prototype. The bottom view shows the treels $\left.{ }^{(}\right)$drive mechanism rotating in respect to the main body.

\section{A. Mobility}

The mobility of the system is ensured by a combination of tracks and wheels. We call this type of structure Differential Treels ${ }^{\complement}$ Drive $^{2}$. Each side of the treels ${ }^{\complement}$ (one track and one wheel) is controlled by a motor so that the s-bot can freely move in the environment and easily rotate on the spot. This structure enable a very good mobility thanks to the position of the wheel and their diameter larger than the tracks height. The treels ${ }^{(c)}$ driving system allow each s-bot to move in moderately rough terrain, with more complex situations being addressed by SWARM-BOT configurations. This kind of modularity and flexibility to pass large obstacles is very similar to the one developed by self-reconfigurable robots [11]. The main difference consists in the fact that the SWARM-BOT has less 3D capabilities than self-reconfigurable robots, an s-bot being able to lift only one s-bot. This aspect is compensated by exploiting the mobility of each module, which is not present in self-reconfigurable robots.

The treels ${ }^{\circledR}$ base can rotate with respect to the main body by means of a motorized axis, as illustrated in figure 2 .

\footnotetext{
${ }^{2}$ Treels ${ }^{\circledR}$ comes from TRacks and whEELS
}

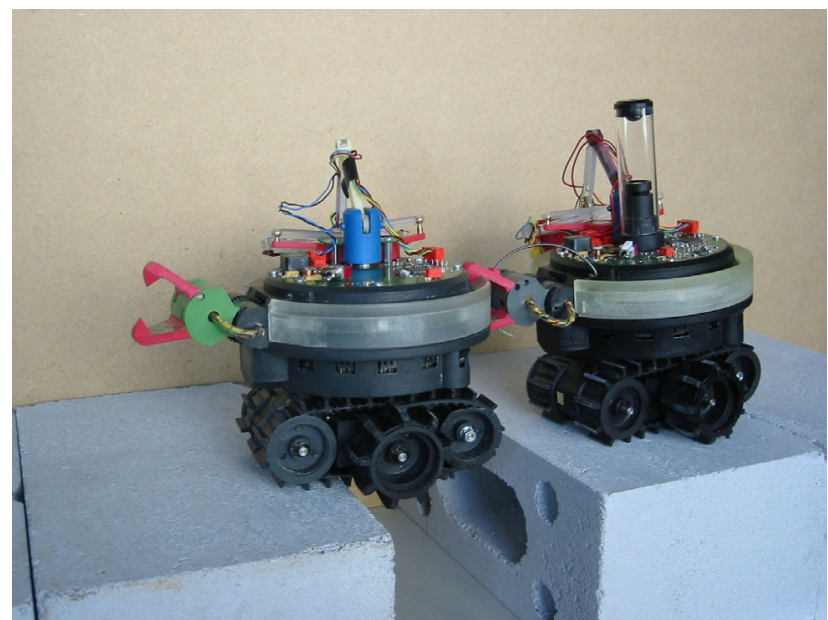

Fig. 3. The front gripper is used to connect in a secure way to other b-bots and form chains to pass large obstacles or holes (see video).

\section{B. Interconnections}

S-bots have two types of possible physical interconnections: rigid and semi-flexible.

Rigid connections (figure 3 ) between s-bots are implemented by a gripper mounted on a horizontal active axis. The gripper can connect to other s-bots on a T-shaped ring around the main s-bot body. If not completely closed, the connection leaves some degrees of freedom, which are very important for positioning and physical interaction between robots. If completely closed, the gripper ensures a rigid connection and can be used to lift other s-bots.

The shape of the gripper enables a very large acceptance area necessary to securely grasp at any angle and lift (if necessary) another s-bot. This is a very important aspect for connections that take place in rough terrain between autonomous robots. It has to be considered that interconnecting robots to build a self-assembling SWARM-BOT is a very different action than interconnecting modules in a self-reconfigurable robot. Self-reconfigurable robots can compute the exact position of each module in order to ensure precise positioning during interconnection [1]. This is not the case in self-assembling robots like the SWARM-BOT where one needs freedom to connect at several angles and in less controlled situations. This feature overcomes the problem of precise position matching that self-reconfigurable robots face.

Semi-flexible connections (figure 4) are implemented by a flexible parallel ${ }^{3}$ arm actuated by three motors positioned at the point of attachment on the main body. The three degrees of freedom allow the arm to extend, move laterally and vertically. The arm ends with a gripper similar to the one used for rigid connections. It connects on the same

\footnotetext{
${ }^{3}$ The term parallel applies to the structure of the arm. See [7] for more details.
} 


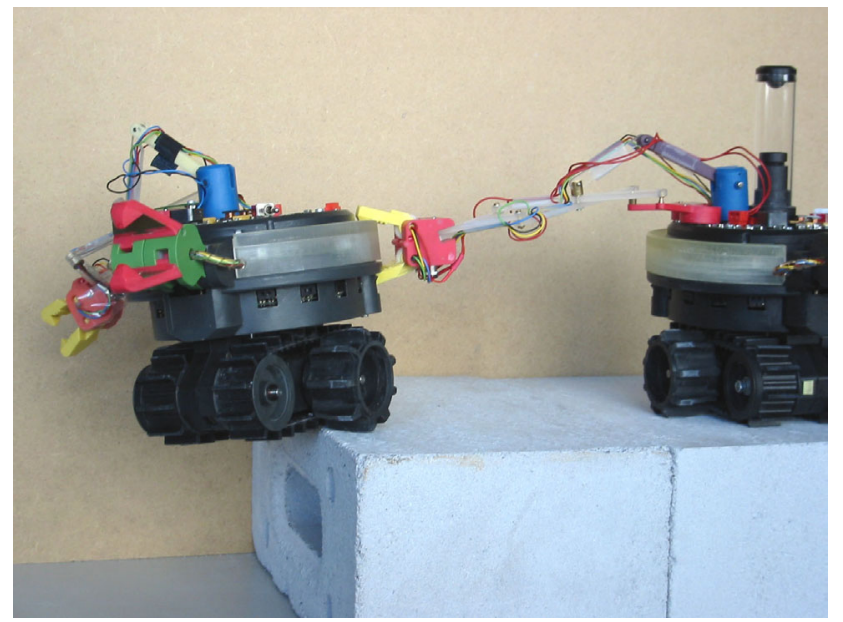

Fig. 4. Lateral semi-flexible connections are used to keep relative mobility between s-bots while they are in a SWARM-BOT configuration. This flexible structure can help for instance to assist another s-bot in unstable situations.

ring as the rigid connection.

Rigid and semi-flexible connections have complementary roles in the functioning of the SWARM-BOT. The rigid connection is mainly used to form rigid chains that have to pass large gaps or obstacles, as illustrated in figure 3. The semi-flexible connection is adapted for configurations where each robot can still have its own mobility inside the structure. An example is illustrated in figure 4. The SWARM-BOT can of course also have mixed configurations, including both rigid and semi-flexible connections. This dual mode of connection is another main characteristics of the SWARM-BOT concept in respect to other self-reconfigurable robots [11].

Both the rigid and flexible grippers can also be used to grasp objects, which is another main difference with self-reconfigurable robots.

\section{Sensors}

Each s-bot is a fully autonomous mobile robot and is equipped with all the sensors necessary for navigation, such as a color omnidirectional camera, 16 lateral and 4 bottom infra-red proximity sensors, 24 light sensors, a 3 axis accelerometer, two humidity sensors as well as incremental encoders and torque sensors on each of the nine degree of freedom. In addition to these basic features, each robot is equipped with sensors and communication devices to detect and communicate with other s-bots. Typical devices implementing these features are again the omnidirectional camera combined with 24 color LEDs all around the robot (inside the transparent T-shaped ring, to express "state" of the robot) and in the grippers, 8 local color detectors all around the body and inside the grippers as well as one speaker and three microphones. Despite the

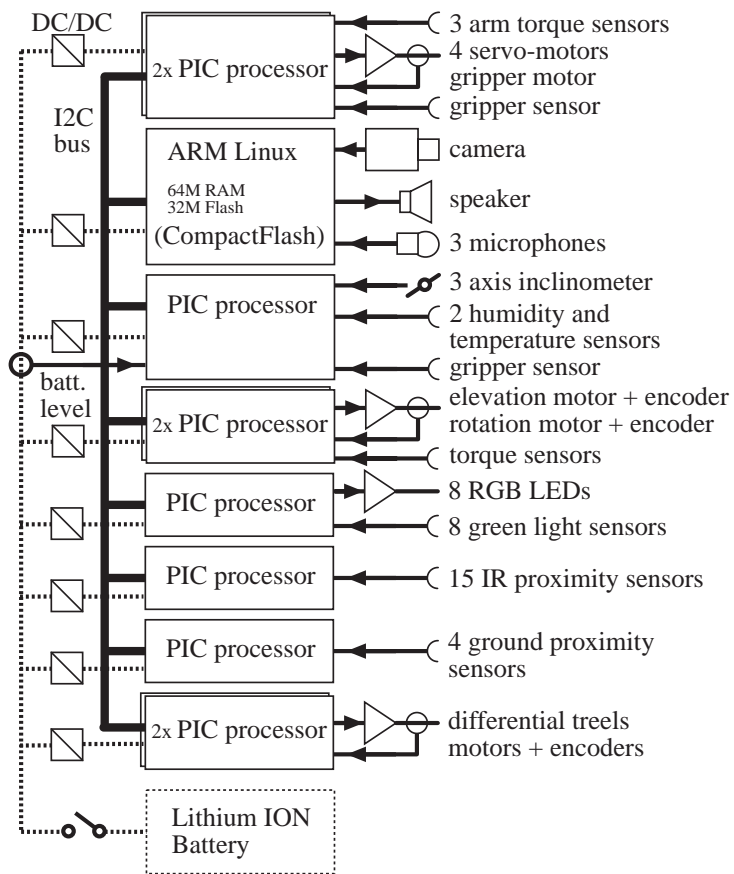

Fig. 5. Electronics structure. Eleven processors share the management of about 50 sensors and 9 actuators.

availability on the robot of a wireless LAN, images and sound will be the only communication channels between s-bots used in our experiments. Radio LAN will be used exclusively for debugging and monitoring of experiments.

In addition to multiple perception modes implemented by several sensors, we made a careful differentiation on range of perception. Research on collective insects [5] shows that collective behaviors are often based on multi-range and multi-modal sensing in order to perceive and exchange signals at multiple levels and in several circumstances. For this reason, as well as for more practical reasons of interferences, infra-red proximity (active) sensors have been limited to a very short range. Sound covers a much longer range and the camera is used both for long and short range sensing, depending on the features extracted from the image.

Placing an autonomous robot as basic component for the modular system is a radically different choice than in selfreconfigurable robots, where the basic element includes only one or two degrees of freedom and very few sensors. This different choice is motivated by the clear goal of distributed autonomous control in the SWARM-BOTS project. In most self-reconfigurable robots the control is implemented in a centralized way or by remote control [11]. Only few groups working on self-reconfigurable robots have presented experiments on distributed control [16], [14]. 


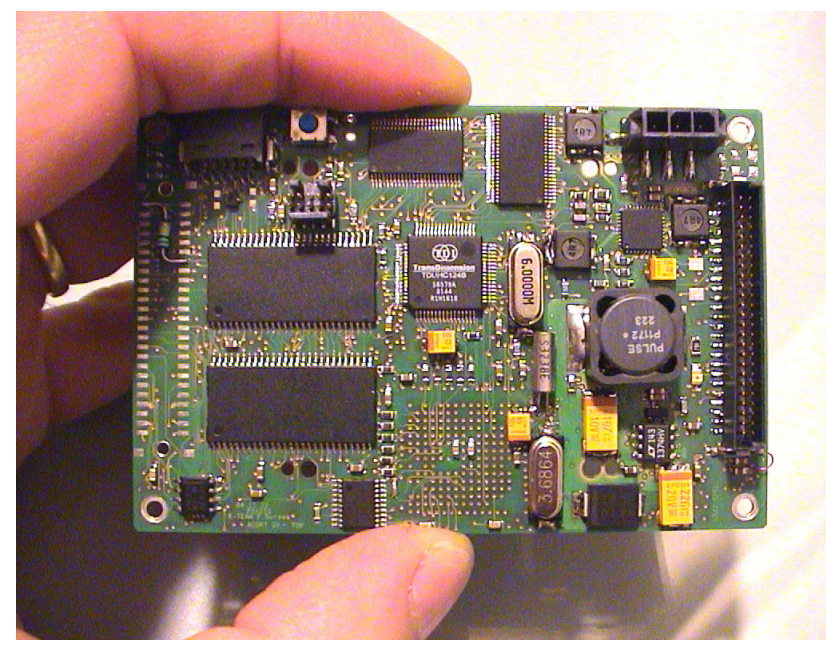

Fig. 6. Main ARM board prototype running LINUX. The processor can run at $400 \mathrm{MHz}$, has $64 \mathrm{M}$ of RAM and $32 \mathrm{M}$ of FLASH, USB, $\mathrm{I}^{2} \mathrm{C}$, RS232 and two CompactFlash slots. The power consumption is $500 \mathrm{~mW}$ at $100 \mathrm{MHz}$ and $700 \mathrm{~mW}$ at $400 \mathrm{MHz}$.

\section{CPU and control electronics}

The control strategy of the SWARM-BOT consists of distributed algorithms based on local information and simple self-organization rules inspired upon ant colony behaviors. Although this type of control algorithm does not need much computational power, the large number of sensors and degrees of freedom requires fast preprocessing and efficient control. The type of experiment that will be conducted on this system, very similar to biological experiments, will also need very good monitoring and collection of large quantity of data for software development and experiment analysis. For all these reasons the s-bots have been equipped with a network of eleven processors (see figure 5), each of them responsible for a sub-task in the system. The most powerful processor, an ARM based processor running LINUX, is in charge of the management of the system, of the processing of the most complex sensors and of the communication with a base station for monitoring purposes.

\section{S-BOT IMPLEMENTATION}

As illustrated in figure 1, a prototype has been implemented including all electronics parts excepted the ARM main board and the connected camera and sound system, which have been prototyped separately (see figure 6). This sections describes aspects of the implementation that make the s-bot suitable for swarm configurations and different from existing robots.

\section{A. Complex miniature mechanics}

Each s-bot is composed of about 100 major parts (see figure 7) and has nine degrees of freedom, most of them equipped with position encoder and torque sensor.

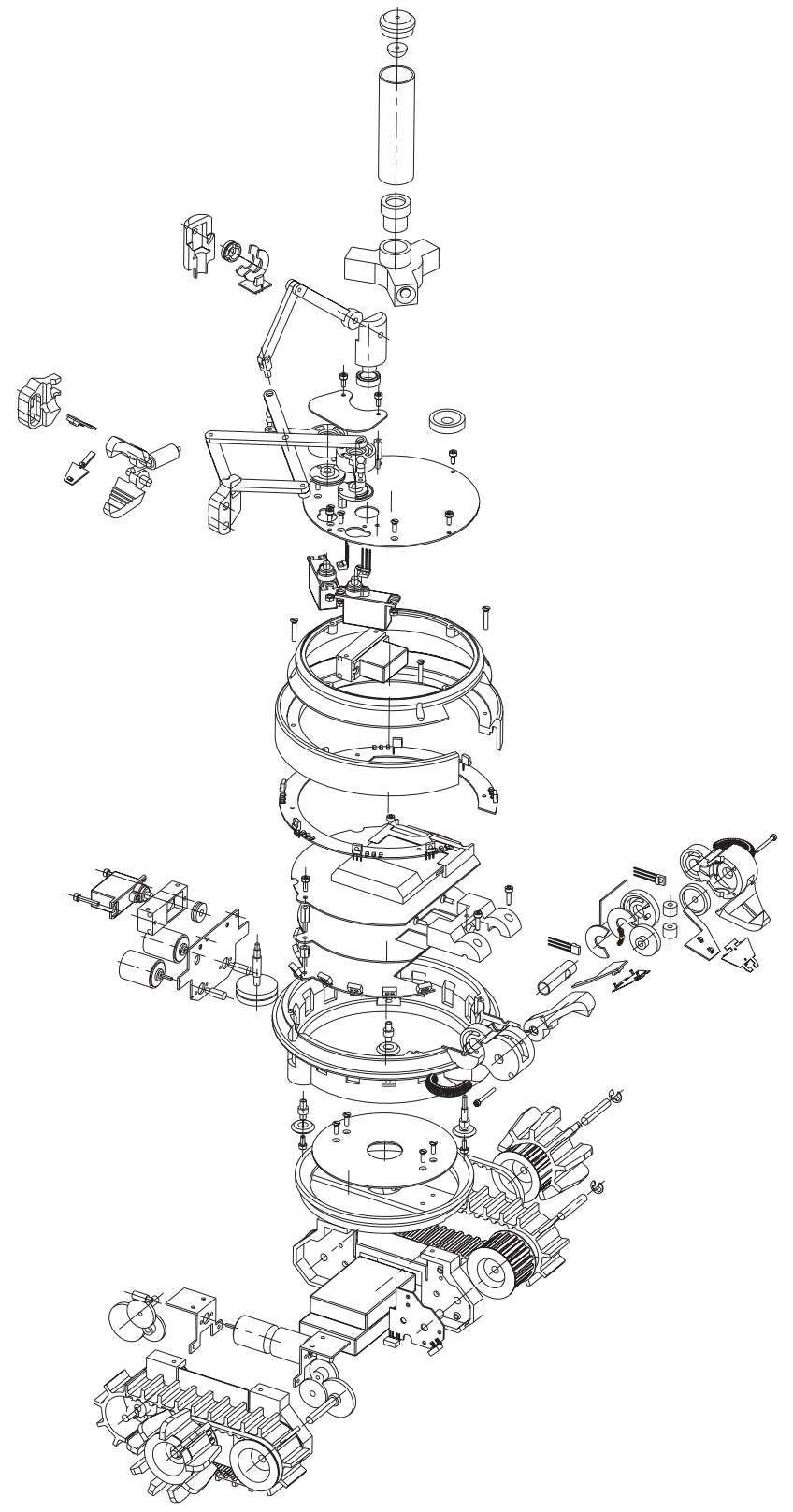

Fig. 7. Mechanical structure. Each s-bot is composed by about 100 major parts.

The main issues addressed here are miniaturization and complexity. Combined, these two factors generate a very dense design, where each half millimeter is used carefully. To correctly address this challenge we have made extensive use of 3D CAD tools, as seen in figures 2 and 7. All details have been designed in 3D, included critical components on the printed circuits. In parallel to CAD design, partial prototyping has been used to verify critical parts or concepts, such as the parallel arm or some aspects of the mechanical torque sensors. Continuous interaction between CAD models and prototyping resulted 

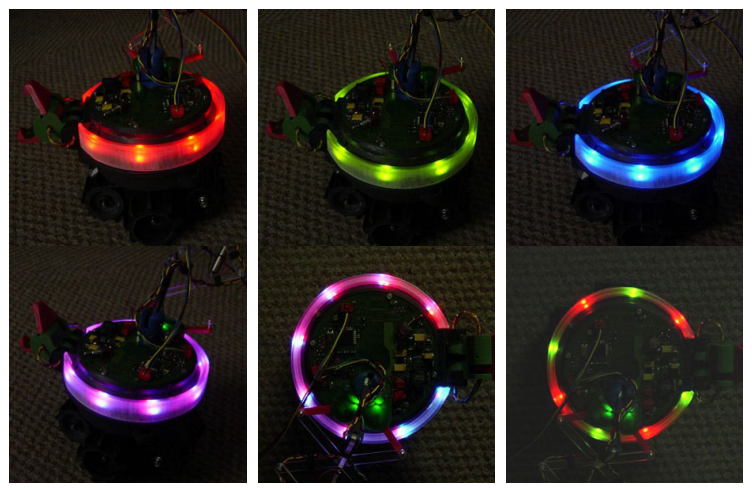

Fig. 8. Some examples of color ring configuration that can be used for sub-symbolic communication among s-bots.

in the design of the first full prototype, which was working perfectly two hours after the first final assembling.

The prototype, which includes most of the electronics, weights only 660 grams. The integration of the main ARM board, camera and sound system will add only some tens of grams, the printed circuits and the connectors being already included in the prototype. We should therefore not exceed 750 grams, which is, for the complexity of the system, very light. This result has been achieved using mainly plastic parts, very thin printed boards and highefficiency miniature motors $(\varnothing 15 \times 16 \mathrm{~mm}, 0.5 \mathrm{~W})$.

\section{B. Distributed electronics}

To control the mechanical system described above, each s-bot needs an efficient electronics, described in figure 5. All ten PIC processors and the related electronics have been included inside the prototype. Only the ARM main board, the video and sound system have been prototyped separately. This last sub-system has the size of a credit card and will be integrated on a printed board already placed within the s-bot. All functionalities of the electronics inside the prototype have been tested and work well. The main elements of the ARM board have been tested and LINUX is running on it.

From the architecture point of view, the system complexity has been addressed using a multi-processor multiprinted circuit structure. The whole electronics has been distributed over 15 printed circuits placed all around in the robot where they are necessary. Cables have been minimized by using print-to-print connectors and having only a serial bus that goes on all main printed circuits, each of them hosting one or several processors. This structure is very similar to the one found on the Khepera robot, developed by Edoardo Franzi in collaboration with some of the authors [13].

\section{High computational power}

The vision and sound sub-systems generate a large amount of data that has to be processed in real time.
Images are processed by color extraction, color position detection and similar simple feature extractions to detect robot states and allow sub-symbolic communication among s-bots (figure 8). Sound is used for simple communication and source orientation extraction. The combination of image and sound processing needs therefore a powerful, but very low power processor. We decided to use the XScale processor from Intel running at $400 \mathrm{MHz}$ and LINUX as operating system. The XScale processor is equipped with $64 \mathrm{M}$ of RAM, 32M of FLASH, USB master and slave interfaces, serial interface, $\mathrm{I}^{2} \mathrm{C}$ bus and two CompactFlash slots. This provides low power consumption (less than $1 \mathrm{~W}$ ) and very good flexibility, both in the choice of extension devices (CompactFlash, USB) and in the choice of programming tool. Figure 6 shows the first XScale board prototype.

\section{Autonomy in energy}

Miniaturization, complex mechanics and powerful electronics impose strong constraints on energy consumption and battery capacity. The s-bot design has been made considering carefully power consumption and battery capacity. At the power consumption level we managed to keep it low by using low power processors and electronics tricks ${ }^{4}$ to limit as much as possible power dissipation. The results is a power consumption between 2.5 and 5 Watts in standard operation, including the main ARM board. This power consumption can be reduced putting the processors in sleep mode when unused. The batteries, placed between the two tracks, have a capacity of $10 \mathrm{Wh}$, ensuring an minimal autonomy of two hours. To achieve this capacity in the small available space we use two Lithium-Ion cells.

\section{CONCLUSION}

This paper presents the SWARM-BOT concept and its implementation in a very compact size. This concept brings innovative elements to bio-inspired and collective robotics, opening new research directions in swarm robotics, collective robotics and distributed intelligence.

Despite the similarity with self-reconfigurable robotics due to the physical connection between s-bots, several key aspects of the SWARM-BOTS approach have shown to be complementary to self-reconfigurable robots. Particularly different are the mobility of each SWARM-BOTS module and their high autonomy, from the point of view of the sensors and the computational power.

Future work will include redesign of the ARM main board to be fitted inside the robot, production of 35 s-bots and experiments of adaptive algorithms in the SWARMBOT configuration.

\footnotetext{
${ }^{4}$ Two examples: the power supply voltage is adapted to the LED voltage to reduce power dissipation, some LEDs are connected in series to better exploit the direct battery voltage.
} 


\section{ACKNOWLEDGMENTS}

Many thanks to Pierre Bureau from K-Team ${ }^{5}$, Stephane Magnenat, Julien Pilet and René Beuchat from the LAPEPFL laboratory ${ }^{6}$ and all the SWARM-BOTS project partners for the inputs, collaboration, suggestions and remarks.

The SWARM-BOTS project is funded by the Future and Emerging Technologies programme (IST-FET) of the European Community, under grant IST-2000-31010. The information provided is the sole responsibility of the authors and does not reflect the Community's opinion. The Community is not responsible for any use that might be made of data appearing in this publication. The Swiss participants to the project are supported under grant 01.0012 by the Swiss Government.

\section{REFERENCES}

[1] S. K. Agrawal, L. Kissner, and M. Yim. Joint solutions of many degrees-of-freedom systems using dextrous workspaces. In Wook H. Kwon et al., editor, Proceedings of the IEEE International Conference on Robotics and Automation, ICRA2001, pages 24802485, Piscataway, NJ, 2001. IEEE. Conference, Seoul, Korea, May 21-26, 2001.

[2] C. Anderson, G. Theraulau, and J.-L. Deneubourg. Self-assemblages in insect societies. Insectes Sociaux, 49:99-110, 2002.

[3] J. Ayers., P. Zavracky, N. McGruer, D. Massa, V. Vorus, R. Mukherjee, and S. Currie. A modular behavioral-based architecture for biomimetic autonomous underwater robots. In Proceedings of the Autonomous Vehicles in Mine Countermeasures Symposium, 1998.

[4] D. Bares, J. an Wettergreen. Dante II: Technical description, results and lessons learned. International Journal of Robotics Research, 18(7):621-649, 1999.

[5] S. Camazine, J.L. Deneubourg, N. Franks, J. Sneyd, E. Bonabeau, and G. Theraulaz. Self-Organisation in Biological Systems. Princeton University Press, 2001.

[6] J. Casper and R. R. Murphy. Issues in intelligent robots for search and rescue. SPIE Ground Vehicle Technology II, 2000.

[7] R. Clavel. Delta, a fast robot with parallel geometry. In Proc. of the International Symposium on Industrial Robots (ISIR), pages 91-100, 1988.

[8] E. Şahin, T.H. Labella, V. Trianni, J.-L. Deneubourg, P. Rasse, D. Floreano, L. Gambardella, F. Mondada, S. Nolfi, and M. Dorigo. SWARM-BOTS: Pattern formation in a swarm of self-assembling mobile robots. In A. El Kamel, K. Mellouli, and P. Borne,

\footnotetext{
${ }^{5}$ http://www.k-team.com

${ }^{6}$ http://lapwww.epfl.ch
}

editors, Proceedings of the IEEE International Conference on Systems, Man and Cybernetics, Hammamet, Tunisia, October 6-9, 2002. Piscataway, NJ: IEEE Press.

[9] T. Estier, Y. Crausaz, B. Merminod, M. Lauria, R. Piguet, and R. Siegwart. An innovative space rover with extended climbing abilities. In Proceedings of Space and Robotics, 2000.

[10] Jacob Fredslund and Maja J. Matarić. Robot Formations Using Only Local Sensing and Control. In Proceedings of IEEE International Symposium on Computational Intelligence for Robotics and Automation (CIRA 2001), Banff, Canada, 29th July-1st August 2001.

[11] A. Kamimura, S. Murata, E. Yoshida, H. Kurokawa, K. Tomita, and S. Kokaji. Self-reconfigurable modular robot - experiments on reconfiguration and locomotion. In T. J. Tarn et al., editor, Proc. of the 2001 IEEE/RSJ International Conference on Intelligent Robots and Systems IROS2001, pages 606612, Piscataway, NJ, 2001. IEEE. Conference, Maui, Hawaii, USA, October 29 - November 3, 2001.

[12] A. Lioni, C. Sauwens, G. Theraulaz, and J.L. Deneubourg. Chain formation in oecophylla longinoda. Journal of Insect Behaviour, 15:679-696, 2001.

[13] F. Mondada, E. Franzi, and P. Ienne. Mobile robot miniaturization: A tool for investigation in control algorithms. In Proceedings of the Third International Symposium on Experimental Robotics, Kyoto, Japan, 1993.

[14] B. Salemi, W.-M. Shen, and P. Will. Hormone controlled metamorphic robots. In Wook H. Kwon et al., editor, Proceedings of the IEEE International Conference on Robotics and Automation, ICRA2001, pages 4194-4199, Piscataway, NJ, 2001. IEEE. Conference, Seoul, Korea, May 21-26, 2001.

[15] H. W. Stone. Mars pathfinder microrover: A lowcost, low-power spacecraft. In Proceedings of the 1996 AIAA Forum on Advanced Developments in Space Robotics, 1996.

[16] K. Støy, W.-M. Shen, and P. Will. Global locomotion from local interaction in self-reconfigurable robots. In Proceedings of the 7th international conference on intelligent autonomous systems (IAS-7), 2002. Conference, Marina del Rey, CA, Mar 25-27, 2002.

[17] G. Visentin, M. Van Winnendael, and P. Putz. Advanced mechatronics in ESA space robotics developments. In T. J. Tarn et al., editor, Proc. of the 2001 IEEE/RSJ International Conference on Intelligent Robots and Systems IROS2001, pages 1261-1266, Piscataway, NJ, 2001. IEEE. Conference, Maui, Hawaii, USA, October 29 - November 3, 2001.

[18] M. Yim, Y. Zhang, and D. Duff. Modular robots. IEEE Spectrum, pages 30-34, February 2002. 